\title{
Factores de riesgo y clasificación de riesgo para enfermedad tromboembólica en mujeres posparto
}

\author{
Mario Santiago Mesa Espinel ${ }^{1,2}$ (D), Mónica Paola Quemba Mesa² (D), Nubia Mercedes González Jiménez² (D), \\ Gina Sofía Montaño Padilla ${ }^{3}$ (D) , Juan David Vega Padilla ${ }^{4}$ (D)
}

\section{RESUMEN}

Introducción. Las mujeres en el periodo posparto presentan un mayor riesgo de morbilidad y mortalidad, con un incremento de hasta 10 veces el riesgo de enfermedad tromboembólica, en comparación con mujeres no embarazadas en edad fértil.

Objetivo. Describir las características sociodemográficas, factores de riesgo y clasificación de riesgo para eventos tromboembólicos de las mujeres posparto en un hospital de referencia del departamento de Boyacá durante el año 2018.

Metodología. Estudio observacional, descriptivo, de corte transversal, realizado en 398 mujeres posparto, quienes fueron encuestadas y valoradas por el servicio de ginecobstetricia del Hospital Regional de Sogamoso, durante el año 2018; se identificaron los factores y se hizo clasificación de riesgo para eventos tromboembólicos.

Resultados y conclusiones. Las mujeres posparto cuentan con una mediana de edad de 26 años [RIQ; 10 años], procedentes del área urbana en el $62,6 \%$ y en su mayoría $(69,6 \%$ ) del estrato socioeconómico uno. Los factores de riesgo más frecuentes encontrados fueron la cesárea $(33,2 \%)$, el sobrepeso $(31,4 \%)$, la obesidad grado I y II $(11,3 \%)$ y el parto pretérmino $(7,5 \%)$. La clasificación de riesgo para eventos tromboembólicos en el total de la población se clasificó como: 6\% riesgo bajo, 89,5\% riesgo moderado y 4,5\% riesgo alto. Es necesario contar en los servicios de ginecobstetricia con la identificación del nivel de riesgo para eventos tromboembólicos de las mujeres posparto, de forma estandarizada y sistemática; igualmente, mitigar estos riesgos y así garantizar la prevención de este tipo de complicaciones.

Palabras claves: factores de riesgo; periodo posparto; trombosis; trombosis venosa.

\footnotetext{
${ }^{1}$ Hospital regional de Sogamoso ESE, Sogamoso, Colombia

2 Universidad de Boyacá, Tunja, Colombia

${ }^{3}$ Medicina Critica y Cuidado Intensivo Universidad de la Sabana, Chía, Colombia

${ }^{4}$ E.S.E. San Jose, Colombia
}

Correspondencia: Juan David Vega Padilla. Correo electrónico: ddavids89@hotmail.com

\section{Citar este artículo así:}

Mesa Espinel MS, Quemba Mesa MP, González Jiménez NM, Montaño Padilla G, Vega Padilla JD. Factores de riesgo y clasificación de riesgo para enfermedad tromboembólica en mujeres pospartoRevista Investig Salud Univ Boyacá. 2020; 7(2). 52-64. doi: https://doi. org/10.24267/23897325.411 
Risk factors and risk classification for thromboembolic disease in postpartum women

\begin{abstract}
Introduction. Women in the postpartum period have a higher risk of morbidity and mortality, with an increase of up to 10 times the risk of thromboembolic disease compared to non-pregnant women of childbearing age.

Objective. To describe the sociodemographic characteristics, risk factors, and risk classification for thromboembolic events of postpartum women in a reference hospital in the department of Boyacá during 2018.

Methodology. Observational, descriptive, cross-sectional study, carried out in 398 postpartum women, who were surveyed and assessed by the Gynecology and Obstetrics Service of the Sogamoso Regional Hospital during 2018, the factors and risk classification for thromboembolic events were identified.

Results and conclusions. Postpartum women have a median age of 26 years [RIQ; 10 years], from the urban area in $62.6 \%$ and mostly from the socioeconomic stratum one with $69.6 \%$. The most frequent risk factors found were caesarean section with $33.2 \%$, overweight with $31.4 \%$, obesity grade I and II with $11.3 \%$ and preterm delivery with $7.5 \%$. The risk classification for thromboembolic events in the total population was classified as: $6 \%$ low risk, $89.5 \%$ moderate risk and $4.5 \%$ high risk. Identification of the level of risk for thromboembolic events in postpartum women is necessary in the gynecology and obstetrics services in a standardized and systematic way, as well as mitigating these risks and thus guaranteeing the prevention of this type of complications.
\end{abstract}

Keywords: risk factors, postpartum period, thrombosis, venous thrombosis. 


\section{Fatores de risco e classificação de risco para doença tromboembólica em mulheres no pós-parto}

\section{RESUMO}

Introdução: Mulheres no período pós-parto apresentam maior risco de morbilidade e mortalidade, com aumento de até 10 vezes o risco de doenças tromboembólicas, em comparação com mulheres não grávidas em idade reprodutiva.

Objetivo: Descrever as características sociodemográficas, fatores de risco e classificação de risco para eventos tromboembólicos em mulheres pós-parto no hospital de referência no departamento de Boyacá durante o ano 2018.

Metodologia: Estudo observacional, des-critivo, transversal, realizado em 398 mulheres pós-parto, que foram inquiridas e avaliadas pelo servi-ço de ginecologia e obstetrícia do Hospital Regional de Sogamoso, durante o ano de 2018; Identifica-ram-se fatores e classificação de risco para eventos tromboembólicos.

Resultados e conclusões: as mulheres no pós-parto têm uma idade mediana de 26 anos [RIQ; 10 anos], $62,6 \%$ da zona urbana e a maioria $(69,6 \%)$ do estrato socioeconômico um. Os fatores de risco mais frequentes encontrados fo-ram cesárea $(33,2 \%)$, sobrepeso $(31,4 \%)$, obesidade graus I e II $(11,3 \%)$ e parto prematuro $(7,5 \%)$. A classificação de risco para eventos tromboembólicos na população total foi classificada em: $6 \%$ de baixo risco, $89,5 \%$ de risco moderado e $4,5 \%$ de alto risco. É necessário contar com os serviços de ginecoobstetrícia com a identificação do grau de risco para eventos tromboembólicos em mulheres pós-parto, de forma padronizada e sistematizada; da mesma forma, mitigar esses riscos e, assim, ga-rantir a prevenção deste tipo de complicações.

Palavras-chave: fatores de risco, período pós-parto, trombose, trombose venosa. 


\section{INTRODUCCIÓN}

En Colombia, las principales causas de mortalidad materna son la preeclampsia, eclampsia, hemorragia posparto, muerte por causa obstétrica no especifica, sepsis puerperal y la embolia obstétrica (1). Los estudios nacionales acerca de la epidemiología de los eventos tromboembólicos en el periodo posparto aún son escasos y no existen registros claros sobre esta condición (2).

Las mujeres en el periodo posparto presentan un mayor riesgo de morbilidad y mortalidad, con un incremento de hasta 10 veces el riesgo de enfermedad tromboembólica venosa (3) en comparación con el riesgo en mujeres no embarazadas en edad fértil; el cual es de uno a cinco casos por 100.000 mujeres por año (4). El riesgo de enfermedad tromboembólica venosa es aún mayor en el periodo del posparto temprano (5), principalmente en los casos donde se realizó cesárea con 2,1 veces más probabilidad de riesgo en comparación con los partos por vía vaginal (6), siendo frecuente la aparición de trombosis venosa profunda y tromboembolismo pulmonar (7).

Al tromboembolismo venoso se le atribuye 1,1 casos de muerte por cada 100.000 partos, lo cual contribuye desde el $1,2 \%$ al $10 \%$ de las muertes maternas; además, un tercio de las trombosis venosas profundas y la mitad de los tromboembolismos pulmonares ocurren en el periodo pos- parto (8). El embarazo se encuentra asociado a estados de hipercoagulabilidad, y en los países desarrollados la principal causa de mortalidad materna se asocia a los eventos tromboembólicos, siendo en su mayoría de origen venoso $(9,10)$.

Diferentes grupos de trabajo y sociedades han desarrollado guías acerca del tema, considerando al tromboembolismo venoso como una causa mayor de morbilidad y mortalidad en el periodo posparto y, también, reconociendo la falta de estudios al respecto (10-12).

Debido a la falta de investigación en este campo, el objetivo de este estudio fue realizar la descripción de las características sociodemográficas, factores de riesgo y clasificación de riesgo para eventos tromboembólicos de las mujeres posparto en un hospital de referencia del centro-oriente del departamento de Boyacá, durante el año 2018.

\section{METODOLOGÍA}

Fue llevado a cabo un estudio observacional y descriptivo, de corte transversal, en el Hospital Regional de Sogamoso, sobre una población de 1600 mujeres en periodo posparto. El tamaño de la muestra fue calculado en el programa Epidat versión 4.1, los parámetros incluidos fueron: precisión del $5 \%$, nivel de confianza al $95 \%$ y una prevalencia esperada del $50 \%$ (esta prevalencia fue calculada debido a la falta de investigaciones que 
determinaran una prevalencia local). Se ajustó el tamaño de la muestra para probables pérdidas del 15\%. A partir de lo anterior, se determinó un tamaño de muestra mínimo de 357 mujeres en posparto.

La recolección de los datos se realizó entre el 1 de marzo y el 31 de octubre del año 2018. Los criterios de inclusión correspondieron a mujeres en posparto, independientemente de su edad, paridad o vía del parto, hospitalizadas durante el tiempo analizado; por otra parte, los criterios de exclusión fueron: mujeres en posparto que no firmaron el consentimiento informado para participar en el estudio o pacientes con alguna condición que impedía la recolección de los datos (condiciones médicas críticas).

\section{Análisis estadístico}

La base de datos fue registrada en Excel versión 2013 y analizada en el "Statistical Package for the Social Sciences" (SPSS) versión 21. Las variables incluidas fueron: variables sociodemográficas (edad, procedencia, estrato socioeconómico), variables antropométricas (índice de masa corporal, estado nutricional) y las variables clínicas (edad gestacional, número de partos, estado nutricional, factores de riesgo como cesárea, sobrepeso, obesidad, parto pretérmino, puerperio con hospitalización mayor a 3 días, várices dolorosas, preeclampsia, diabetes mellitus, procedimiento quirúrgico en embarazo o puerperio, tabaquismo, hipertensión arterial crónica, hemorragia posparto, transfusión mayor a 2 unidades de glóbulos rojos, óbito, hiperémesis gravídica, evento tromboembólico previo, embarazo múltiple, sepsis durante el embarazo, hemorragia ante-parto, síndrome de hiperestimulación ovárica, insuficiencia renal previa, trombofilia y enfermedad inflamatoria intestinal, además de la clasificación de riesgo bajo, moderado, alto y muy alto).

Los factores de riesgo y la clasificación fueron tomados de la "Guía de práctica clínica para la prevención de eventos tromboembólicos venosos durante la gestación, el parto o el puerperio" para Colombia(12). Las variables de tipo categórico, por ejemplo, la procedencia, el estrato socioeconómico, etc., fueron descritas con frecuencias absolutas (recuento) y frecuencias relativas (\%), para ser representadas en tablas con su respectiva interpretación, las variables de tipo numérico, por ejemplo, la edad, el índice de masa corporal, etc., fueron analizadas con el método estadístico de Kolmogorov Smirnov, el cual permitió determinar que las variables numéricas de este estudio no cumplían el supuesto de normalidad (valor $p<0,05$ ) y fueron representadas por medio de medidas de dispersión, posición y de tendencia central correspondientes con estadística no paramétrica (rango intercuartil, percentiles y mediana). 


\section{Consideraciones éticas}

Según la resolución 8430 de 1993 este estudio fue clasificado como una investigación sin riesgo. Todas las pacientes que ingresaron al estudio realizaron la firma de consentimiento informado. El trabajo de investigación fue aprobado por el comité de Bioética de la universidad de Boyacá según el memorando CB 423.

\section{RESULTADOS}

En total, ingresaron al estudio 398 mujeres atendidas durante el año 2018 en el Hospital Regional de Sogamoso; con esa cantidad se cumplió de forma adecuada el tamaño de muestra, la cual fue representativa de la población de 1600 mujeres.

\section{Variables sociodemográficas}

La tabla 1 describe las características sociodemográficas de las mujeres en posparto. Con respecto a la edad, en este grupo de mujeres se observa una mediana de 26 años, una edad mínima de 15 años y una edad máxima de 45 años. La procedencia fue urbana $(62,6 \%)$ y, en su mayoría, pertenecían al estrato socioeconómico uno $(69,6 \%)$.

población, su índice de masa corporal, el estado nutricional pregestacional, la paridad y la edad gestacional en las mujeres posparto, se tienen los siguientes datos: en esta población el índice de
Tabla 1. Características sociodemográficas en las mujeres posparto.

\begin{tabular}{cc}
\hline Variable sociodemográfica & Resultado \\
\hline & $\mathbf{n}=\mathbf{3 9 8}$ \\
\hline Edad en años & $26(10)^{*}$ \\
& Q1 21 \\
Procedencia & Q3 31 \\
\hline & Urbana: $249(62,6 \%)$ \\
\hline Estrato socioeconómico & Rural: $149(37,4 \%)$ \\
\hline & Uno: $277(69,6 \%)$ \\
& Dos: $115(28,9 \%)$ \\
& Tres: $1(0,3 \%)$ \\
\hline
\end{tabular}

* Mediana (Rango intercuartil) Fuente: Autores.

En relación con las características clínicas de esta masa corporal tuvo una mediana de 24,2 , la clasificación nutricional pregestacional más frecuente fue peso normal con $54 \%$, la mayoría fueron primigestantes con $44,2 \%$, la mediana de edad gestacional fue de 39,1 semanas, con una edad gestacional mínima de 30,5 semanas y una edad gestacional máxima de 40,5 semanas.

Con respecto a los factores de riesgo para eventos tromboembólicos en mujeres posparto según la Guía colombiana de práctica clínica para prevención de eventos tromboembólicos durante la gestación, parto y puerperio (12), los principales factores de riesgo encontrados en las mujeres 
posparto fueron: la cesárea, el sobrepeso, la obesidad grado I y II, el parto pretérmino, puerperio con hospitalización mayor a 3 días, várices dolorosas y preeclampsia. No se presentaron casos de trombofilias (déficit de proteína $S$, déficit de proteína $C$, déficit de antitrombina III, factor $V$ de Leiden), lupus eritematoso sistémico (LES), dermatomiositis, artritis reumatoidea, sepsis puerperal o de corioamnionitis.

La tabla 2 muestra la clasificación del riesgo para eventos tromboembólicos en mujeres posparto. La clasificación de riesgo fue tomada de la Guía colombiana de práctica clínica para prevención de eventos tromboembólicos durante la gestación, parto y puerperio (12); se observó a la clasificación de riesgo moderado como la más frecuente con un $89,5 \%$ y ningún paciente fue categorizado como riesgo muy alto.

Tabla 2. Clasificación del riesgo de eventos tromboembólicos según la Guía colombiana de práctica clínica para prevención de eventos tromboembólicos durante la gestación, parto y puerperio.

\begin{tabular}{cc}
\hline Clasificación de riesgo & $\begin{array}{c}\text { Resultado } \\
\mathbf{n}=\mathbf{3 9 8}\end{array}$ \\
\hline Riesgo bajo & $24(6 \%)$ \\
\hline Riesgo moderado & $356(89,5 \%)$ \\
\hline Riesgo alto & $18(4,5 \%)$ \\
\hline Riesgo muy alto & $0(0 \%)$ \\
\hline
\end{tabular}

Fuente: Autores
La tabla 3 describe la clasificación de riesgo de eventos tromboembólicos en mujeres posparto según la procedencia. Entre las mujeres posparto rurales y urbanas la clasificación de riesgo más frecuente fue moderada con $88,8 \%$ y $90,6 \%$ respectivamente. Las mujeres de riesgo alto presentaron una mayor frecuencia en el grupo proveniente del área rural con $4,8 \%$ en comparación con las mujeres del área urbana con $4 \%$. Asimismo, en la tabla 3 se muestra la clasificación de riesgo de eventos tromboembólicos en mujeres posparto según el estrato socioeconómico. La mayoría de mujeres se ubican en los estratos 1 y 2 , donde se observa una mayor frecuencia en la clasificación de riesgo alto en comparación con los estratos 3 y 4 . Los resultados de clasificación de riesgo en el estrato 5 presentan una frecuencia del 33,3\% para cada clasificación de riesgo (bajo, moderado y alto), sin embargo; esta puede estar influenciada por el reducido número de participantes del estrato 5 .

Tabla 3. Clasificación de riesgo de eventos tromboembólicos en mujeres posparto según procedencia y estrato socioeconómico.

\begin{tabular}{ccc}
\hline \multicolumn{3}{c}{ Clasificación según la procedencia. } \\
\hline $\begin{array}{c}\text { Clasificación de } \\
\text { riesgo }\end{array}$ & Rural & Urbana \\
\hline Riesgo bajo & $\mathrm{n}=249$ & $\mathrm{n}=149$ \\
\hline Riesgo moderado & $16(6,4 \%)$ & $8(5,4 \%)$ \\
\hline Riesgo alto & $221(88,8 \%)$ & $135(90,6 \%)$ \\
\hline
\end{tabular}




\begin{tabular}{|c|c|c|c|c|c|}
\hline \multicolumn{6}{|c|}{ Clasificación según la procedencia. } \\
\hline \multicolumn{2}{|c|}{ Riesgo muy alto } & \multicolumn{2}{|c|}{$0(0 \%)$} & \multicolumn{2}{|c|}{$0(0 \%)$} \\
\hline \multicolumn{6}{|c|}{ Clasificación según el estrato socioeconómico } \\
\hline \multirow{3}{*}{$\begin{array}{l}\text { Clasifi- } \\
\text { cación de } \\
\text { riesgo }\end{array}$} & \multirow{3}{*}{$\begin{array}{l}\text { Estra- } \\
\text { to } 1 \\
n=277\end{array}$} & \multirow{3}{*}{$\begin{array}{l}\text { Estra- } \\
\text { to } 2 \\
\mathrm{n}=115\end{array}$} & \multirow{3}{*}{$\begin{array}{c}\text { Estrato } \\
\begin{array}{c}3 \\
\mathrm{n}=1\end{array}\end{array}$} & \multirow{3}{*}{$\begin{array}{c}\text { Estrato } \\
\begin{array}{c}4 \\
\mathrm{n}=2\end{array}\end{array}$} & \multirow{3}{*}{$\begin{array}{c}\text { Estra- } \\
\text { to } 5 \\
n=3\end{array}$} \\
\hline & & & & & \\
\hline & & & & & \\
\hline $\begin{array}{l}\text { Riesgo } \\
\text { bajo }\end{array}$ & $\begin{array}{c}18 \\
(6,5 \%)\end{array}$ & $\begin{array}{c}5 \\
(4,3 \%)\end{array}$ & $0(0 \%)$ & $0(0 \%)$ & $\begin{array}{c}1 \\
(33,3 \%)\end{array}$ \\
\hline $\begin{array}{c}\text { Riesgo } \\
\text { modera- } \\
\text { do }\end{array}$ & $\begin{array}{c}248 \\
(89,5 \%)\end{array}$ & $\begin{array}{c}104 \\
(90,4 \%)\end{array}$ & $\begin{array}{c}1 \\
(100 \%)\end{array}$ & $\begin{array}{c}2 \\
(100 \%)\end{array}$ & $\begin{array}{c}1 \\
(33,3 \%)\end{array}$ \\
\hline $\begin{array}{c}\text { Riesgo } \\
\text { alto }\end{array}$ & $11(4 \%)$ & $6(5,2 \%)$ & $0(0 \%)$ & $0(0 \%)$ & $\begin{array}{c}1 \\
(33,3 \%)\end{array}$ \\
\hline $\begin{array}{c}\text { Riesgo } \\
\text { muy alto }\end{array}$ & $0(0 \%)$ & $0(0 \%)$ & $0(0 \%)$ & $0(0 \%)$ & $0(0 \%)$ \\
\hline
\end{tabular}

Fuente: Autores.

\section{Discusión}

El tromboembolismo venoso es una de las principales causas de morbilidad y mortalidad en gestantes (13), siendo más común en el embarazo y periodo posparto (14), debido al estado de hipercoagulabilidad que acompaña de forma fisiológica a la gestación como prevención de la hemorragia posparto (15). Múltiples factores de riesgo han sido identificados en el puerperio como causas de eventos trombóticos (16), entre estos se puede observar: un índice de masa corporal mayor a 25, inmovilidad, parto pretérmino, preeclampsia, hipertensión arterial, cesárea, infección posparto y hemorragia posparto $(13,17)$.
Los datos descritos son similares a los hallazgos de la presente investigación, porque se identificaron factores de riesgo en mujeres posparto como cesárea, sobrepeso/obesidad, parto pretérmino y preeclampsia. La gestación y el puerperio están relacionados con cambios fisiológicos que favorecen a la formación de estados trombóticos (18), entre estos cambios se han descrito; el aumento de los factores de la coagulación II, VII, VIII y X, incremento en la cantidad de fibrina, disminución de la actividad fibrinolítica y disminución en la actividad de las proteínas S y C (19).

En esta investigación no se observó ningún caso de trombofilia como factor de riesgo en el periodo del puerperio, por lo cual se puede decir que aunque se encuentran descritos los estados de trombofilia en la literatura (20), no son un factor de riesgo frecuente en las mujeres posparto atendidas en el Hospital Regional de Sogamoso. Otros autores han descrito un grupo adicional de factores de riesgo para eventos tromboembólicos en mujeres posparto como (21): historia personal de trombosis, procedimientos quirúrgicos durante el embarazo o el puerperio, más de 3 partos, edad mayor a 35 años, síndrome de hiperestimulación ovárica y enfermedad inflamatoria intestinal $(12,21)$; por otra parte, en los hallazgos de las mujeres posparto del Hospital Regional de Sogamoso la población tenía una mediana de edad de 26 años y solo el 4,9\% presentó más de 3 partos, el $1,5 \%$ cursó con algún procedimiento quirúrgico 
y el 0,3\% presentó síndrome de hiperestimulación ovárica y enfermedad inflamatoria intestinal, por lo cual se puede decir que estos factores de riesgo presentan una baja frecuencia en las mujeres posparto incluidas en este estudio.

En la publicación de Ferrer (22) se describen factores de riesgo similares a los identificados por Lussana y Clark $(13,21)$, pero como hallazgos adicionales se presentan comorbilidades médicas tipo: várices, enfermedad cardiaca, hipertensión arterial crónica y la muerte fetal (22), en esta investigación se identificó una baja frecuencia de estos factores de riesgo, como la presencia de enfermedad varicosa en el $2,5 \%$, la hipertensión arterial crónica en el $1,5 \%$ y el óbito en el $0,8 \%$.

Según Gray et al (23) las mujeres con eventos tromboembólicos previos tienen una mayor recurrencia de eventos trombóticos durante el embarazo y el puerperio, la tasa de recurrencia de trombosis para mujeres con un solo evento trombótico previo durante el puerperio es del $10 \%$ (23); estos datos difieren de los resultados aquí publicados, puesto que la presencia de evento trombótico previo solo ocurrió en el $0,5 \%$ de las mujeres posparto, sin registrar algún episodio de trombosis adicional.

En el estudio de Navas se describe al tabaquismo, la enfermedad autoinmune/enfermedad del colágeno y el embarazo múltiple como factores de riesgo para enfermedad tromboembólica (14), los cuales también fueron registrados en los resultados de este proyecto de investigación, donde la frecuencia de tabaquismo fue del 1,5\%, el embarazo múltiple estaba presenten en el $0,5 \%$ y no se registraron casos de enfermedad del colágeno o enfermedad autoinmune.

En un estudio publicado en Cuba, se describen como factores de riesgo para eventos trombóticos en el posparto a la hiperémesis gravídica y la transfusión, datos que concuerdan con los resultados de este trabajo porque se observó a la hiperémesis gravídica en el $0,8 \%$ de la población y la transfusión de más de 2 unidades de glóbulos rojos en el 1,3\% (24).

Nápoles et al. describe al reposo prolongado (25) y Montaño et al. a la infección (26) como factores de riesgo para desarrollar eventos tromboembólicos venosos; en la presente investigación se describió la prevalencia de hospitalización en puerperio por más de 3 días en el 3\% y la sepsis puerperal en el $0,5 \%$ de las mujeres posparto.

Recientemente la Federación Colombiana de Ginecología y Obstetricia publicó la Guía de prevención para eventos tromboembólicos venosos durante la gestación, el parto y el puerperio (12), la cual constituye el principal referente de este trabajo; de esta forma, se logró realizar la primera descripción de factores de riesgo y clasi- 
ficación de riesgo para eventos tromboembólicos en mujeres posparto según esta última actualización de la guía colombiana, ubicando las mujeres posparto en 4 grupos: riesgo bajo $6 \%$, riesgo moderado $89,5 \%$, riesgo alto $4,5 \%$ y riesgo muy alto $0 \%$.

Un estudio de similares características, realizado en 3 hospitales de Colombia, clasificó el riesgo de las pacientes para eventos tromboembólicos en bajo, medio y alto, según la guía del Royal College Obstetrics \& Gynecology; en ese caso, el $1,9 \%$ se clasificó como alto riesgo, el $42 \%$ como riesgo medio y el $56,1 \%$ como riesgo bajo (1), con lo cual se puede observar una diferencia sustancial en los resultados de la clasificación de riesgo, tal vez porque las guías del Royal College fueron diseñadas para población europea, su clasificación de riesgo fue estratificada en tres niveles, diferente a la guía colombiana que se encuentra conformada por 4 categorías o por diferencias epidemiológicas implícitas de los hospitales de referencia en las cuales fueron aplicadas dichas recomendaciones.

Este estudio, de corte transversal, tiene una validez interna adecuada porque cumple de forma apropiada con el tamaño de muestra y pueden generalizarse sus resultados a la población atendida en el Hospital Regional de Sogamoso. Con respecto a la validez externa, este estudio es la primera descripción de factores de riesgo y clasificación de riesgo en el departamen- to de Boyacá, según la última actualización de la guía colombiana para la prevención para eventos tromboembólicos venosos durante la gestación, el parto y el puerperio, por lo cual podría ser un referente para otros estudios en el territorio nacional. Con respecto a las limitaciones del estudio, se puede nombrar los sesgos propios de los diseños de corte transversal y también la falta de un muestreo probabilístico.

\section{Conclusiones}

Esta investigación tenía como objetivo describir las variables sociodemográficas, los factores de riesgo y la clasificación de riesgo para eventos tromboembólicos en mujeres posparto de un hospital de referencia en el departamento de Boyacá; con ese fin, se logró establecer que la mayoría de las participantes son mujeres jóvenes, procedentes del área urbana y con un estrato socioeconómico bajo, además, se identificó que dentro de los factores de riesgo ya conocidos en la guía colombiana los más comunes en esta población fueron cesárea, obesidad/sobrepeso y parto pretérmino. Cuando se clasificó el nivel de riesgo para eventos tromboembólicos la mayoría de las mujeres en posparto tenían un riesgo moderado con $89,5 \%$.

\section{Declaración de conflictos de interés}

Los autores declaran no tener conflicto de intereses. 


\section{Financiación}

El estudio fue financiado en su totalidad por la Universidad de Boyacá.

\section{Bibliografía}

1. Silva-Herrera JL, Duque-Giraldo MA, Torres-Chaparro C, Sanín-Blair JE, Niño-Peña ML, Higuita-Rendón AM. Profilaxis tromboembólica en pacientes posparto. Estudio de corte transversal en tres instituciones en Colombia. Rev Colomb Obstet Ginecol, 2016; 67(1):2025. http://dx.doi.org/10.18597/rcog.364

2. Álvarez GAV, Jaramillo BA, Vélez LIG. Mortalidad materna por fenómenos tromboembólicos en el departamento de Antioquia, años 2004-2005. La salud de las madres en Antioquia: un reto, un derecho, un compromiso, p183. 2007

3. Reina Gutiérrez L, Carrasco Carrasco JE. Recomendaciones sobre profilaxis, diagnóstico y tratamiento de la enfermedad tromboembólica venosa en atención primaria. Resumen del Documento de consenso SEACV-SEMERGEN. Angiología. 2015; 67(5):399-408. https://doi. org/10.1016/j.angio.2015.02.001

4. Barco S, Nijkeuter M, Middeldorp S. Pregnancy and venous thromboembolism. Semin Thromb
Hemost. 2013; 39(05): 549-558. https://doi. org/10.1055/s-0033-1343893

5. Silva Reyna P. Enfermedad tromboembólica venosa en el embarazo. Rev Mex Anest. 2020; 43(1):41-47. http://dx.doi.org/10.35366/ CMA201G.

6. James $A H$, Jamison MG, Brancazio LR, Myers ER. Venous thromboembolism during pregnancy and the postpartum period: incidence, risk factors, and mortality. Am J Obstet Gynecol 2006; 194(5):1311-1315. https://doi. org/10.1016/j.ajog.2005.11.008

7. Tooher R, Gates S, Dowswell T, Davis U. Prophylaxis for venous thromboembolic disease in pregnancy and the early postnatal period. Cochrane Database Syst Rev, 2010. (5):CD001689 https:// doi.org/10.1002/14651858.CD001689.pub2

8. Psbinger I, Ay C. V Biomarkers and Venous Thromboembolism. Arterioscler Thromb Vasc Biol. 2009;29:332-336: https://doi. org/10.1161/ATVBAHA.108.182188

9. James $A H$. Pregnancy and thrombotic risk. Crit Care Med. 2010; 38:S57-S63. https://doi. org/10.1097/CCM.0b013e3181c9e2bb

10. Mclintock, C., Brighton, T., Chunilal, S., Dekker, G., Mcdonnell, N., Mcrae,S. Recommenda- 
tions for the diagnosis and treatment of deep venous thrombosis and pulmonary embolism in pregnancy and the postpartum period. Aust Nz J Obstet Gyn, 2012; 52(1):14-22. https:// doi.org/10.1111/j.1479-828X.2011.01361.x

11. Linnemann $B$, Scholz $U$, Rott $H$, Halimeh $S$, Zotz R, Gerhardt A et al. Treatment of pregnancy-associated venous thromboembolismposition paper from the working Group in Women's health of the Society of Thrombosis and Haemostasis (GTH). Vasa, 2016;45(2), 103-18. https://doi.org/10.1024/0301-1526/ a000504

12. Asociación Bogotana de Obstetricia y Ginecología - Comité de Mortalidad Materna GD de la G de PC, Grillo-Ardila CF, Mogollón-Mariño AC, Amaya-Guío J, Molano-Franco D, et al. Guía de práctica clínica para la prevención de eventos tromboembólicos venosos durante la gestación, el parto o el puerperio. Rev Colomb Obstet Ginecol, 2017;68(4):286-304. Doi: https://doi.org/10.18597/rcog.3084

13. Lussana F, Coppens M, Cattaneo M, Middeldorp $S$. Pregnancy-related venous thromboembolism: risk and the effect of thromboprophylaxis. Thromb Res, 2012; 129(6):673-680. https://doi.org/10.1016/j. thromres.2012.01.017
14. Navas AM, Pérez A. Tromboembolia pulmonar en el embarazo y puerperio: una patología mortal. Medicina Interna, 2017; 33(4).

15. Sucker C. Prophylaxis and therapy of venous thrombotic events (VTE) in pregnancy and the postpartum period. Geburtshilfe und Frauenheilkunde. 2020; 80(01):48-59. https://doi. org/10.1055/a-1030-4546

16. Silvis $S M$, Lindgren $E$, Hiltunen $S$, Devasagayam S, Scheres $\amalg$, Jood K, et al. Postpartum period is a risk factor for cerebral venous thrombosis: a case-control study. Stroke. 2019; 50(2):501-503. https://doi.org/10.1161/ STROKEAHA.118.023017

17. Scheres LJ, Lijfering WM, Groenewegen NFM, Koole S, de Groot CJM, Middeldorp S, et al. Hypertensive complications of pregnancy and risk of venous thromboembolism. Hypertension, 2020;75(3):781-787. https://doi. org/10.1161/HYPERTENSIONAHA.119.14280

18. Rugeri L, Desage S, Khouatra L, Battie C, Doret-Dion M, Trzeciak $C$. et al. Pregnancy-related thrombosis risk in patients with protein $\mathrm{C}$ deficiency and comparison with pregnant women with heterozygous factor $\mathrm{V}$ Leiden mutation. Blood Coagul Fibrinolysis. 2020； 31(1):55-59. https://doi.org/10.1097/ MBC. 0000000000000878 
19. Marik PE. Venous thromboembolism in pregnancy. Clin Chest Med, 2010, 31(4):731-40. https://doi.org/10.1016/j.ccm.2010.06.004.

20. Bistervels IM, Scheres $\sqcup$, Hamulyák EN. Sex matters: Practice 5P's when treating young women with venous thromboembolism. J Thromb Haemost. 2019; 17(9):1417-1429. https://doi.org/10.1111/jth.14549

21. Clark P. Maternal venous thrombosis. Eur J Obstet Gynecol. Reprod Biol. 2008; 139(1):3-10. https://doi.org/10.1016/j. ejogrb.2008.02.011

22. Ferrer MF, Oyarzún EE. Trombosis venosa en el embarazo. Rev méd clín los Condes, 2014, 25(6):1004-1018. https://doi.org/10.1016/ S0716-8640(14)70650-9.

23. Gray G, Nelson-Piercy C. Thromboembolic disorders in obstetrics. Best Pract Res Clin Obstet Gynaecol, 2012, 26(1):53-64. https://doi.org/10.1016/j.bpobgyn.2011.10.003

24. Méndez DN, Núñez DC. Enfermedad tromboembólica venosa en el embarazo y puerperio. Enfoque de riesgo y diagnóstico. Medisan, 2011; 15(10):1423-1444.

25. Nápoles MD, Couto ND. Experiencia de 11 años en la enfermedad tromboembólica venosa en el período grávido puerperal. Rev Cubana Obstet Ginecol, 2011; 37(3):300-311

26. Montaño GS, Quemba MP, González-Jiménez $\mathrm{N}$ et al. Tromboprofilaxis durante el embarazo. Rev Fac Med, 2020; 68 (2). https://doi. org/10.15446/revfacmed.v68n2.73366 